\title{
A Mig-14-like protein (PA5003) affects antimicrobial peptide recognition in Pseudomonas aeruginosa
}

\author{
Nicholas Jochumsen, Yang Liu, Søren Molin and Anders Folkesson \\ Center for Systems Microbiology, DTU-Systems Biology, Building 301, Technical University of \\ Denmark, DK-2800 Lyngby, Denmark
}

\begin{abstract}
Correspondence
Anders Folkesson

anf@bio.dtu.dk
\end{abstract}

Received 7 March 2011

Revised 10 June 2011

Accepted 19 June 2011
The evolution of antibiotic resistance in pathogenic bacteria is a growing global health problem which is gradually making the treatment of infectious diseases less efficient. Antimicrobial peptides are small charged molecules found in organisms from the complete phylogenetic spectrum. The peptides are attractive candidates for novel drug development due to their activity against bacteria that are resistant to conventional antibiotics, and reports of peptide resistance are rare in the clinical setting. Paradoxically, many clinically relevant bacteria have mechanisms that can recognize and respond to the presence of cationic antimicrobial peptides (CAMPs) in the environment by changing the properties of the microbial surface thereby increasing the tolerance of the microbes towards the peptides. In Pseudomonas aeruginosa an essential component of this inducible tolerance mechanism is the lipopolysaccharide modification operon arnBCADTEFPA3559 which encodes enzymes required for LPS alterations leading to increased antimicrobial peptide tolerance. The expression of the operon is induced by the presence of CAMPs in the environment but the molecular mechanisms underlying the cellular recognition of the peptides are poorly elucidated. In this work, we investigate the factors influencing arnB expression by transposon mutagenesis and arnB promoter green fluorescent protein reporters. We have identified a novel gene encoding a Mig-14-like protein that is required for recognition of the CAMPs colistin and Novispirin G10 by P. aeruginosa. Moreover, we show that this gene is also required for the formation of CAMP-tolerant subpopulations in $P$. aeruginosa hydrodynamic flow chamber biofilms.

\section{INTRODUCTION}

Antimicrobial peptides are an integral part of the host defence against infection and have been found in most multi-cellular organisms (Zasloff, 2002). The peptides are small, often cationic and structurally diverse and display broad-spectrum antimicrobial activity against a range of bacteria (Brogden, 2005). Charge interaction between the cationic peptide and the anionic microbial membrane is thought to be a critical step in a process leading to microbial killing (Brogden, 2005). A number of other peptide activities have been identified, indicating roles as multifunctional mediators of immunity, inflammation and wound repair (Hiemstra et al., 2004). Cationic antimicrobial peptides (CAMPs) are evident alternatives for drug

\footnotetext{
Abbreviations: CAMP, cationic antimicrobial peptide; CLSM, confocal laser scanning microscopy; FACS, fluoresence activated cell sorting; GFP, green fluorescent protein; L-Ara4N, 4-amino-4-deoxy-L-arabinose; LPS, lipopolysaccharide; PI, propidium iodide; TCS, two-component system.

Four supplementary tables and three supplementary figures are available with the online version of this paper.
}

development due to their activity on bacteria resistant to conventional antibiotics (Hiemstra et al., 2004). The polymyxins such as colistin are cyclic CAMPs which are highly active against many Gram-negative bacteria and are extensively used in the treatment of Pseudomonas aeruginosa lung infections in patients with cystic fibrosis (Frederiksen et al., 1997; Hoeprich, 1970; Littlewood et al., 1985; Storm et al., 1977). The appearance of bacteria resistant to most classes of antibiotics and the shortage of novel antimicrobial agents with activity against Gramnegative micro-organisms have led to the reemergence of polymyxins as a valuable addition to the drugs available for antimicrobial chemotherapy (Conly \& Johnston, 2006; Landman et al., 2008). Novispirin G10 is an 18 residue linear CAMP which have an amphipathic and $\alpha$-helical coil structure (Travis et al., 2000). The peptide belongs to a large structural family of CAMPs called cathelicidins. Novispirin G10 has been derived from Ovisprin-1 by substituting the tenth amino acid from isoleucine to glycine, which makes Novispirin G10 a less cytotoxic alternative to Ovispirin-1 (Khandelia \& Kaznessis, 2005; Sawai et al., 2002; Steinstraesser et al., 2002). 
It has been suggested that resistance evolution towards antimicrobial peptides is rare, since the peptides exploit such fundamental features of microbial physiological organization that resistance evolution would require changes that are mechanistically impossible, or bestow such a high biological fitness cost that the resistance mutation would not increase in frequency in bacterial populations (Høiby et al., 2001; Perron et al., 2006; Zasloff, 2002). Interestingly, a number of Gram-negative bacteria such as Salmonella enterica and $P$. aeruginosa have specific mechanisms that increase their tolerance (adaptive non-mutational resistance) towards antimicrobial peptides (Conrad \& Galanos, 1989; Vaara et al., 1981). In P. aeruginosa, one of the major mechanisms for tolerance is remodelling of the outer membrane by addition of 4-amino-4-deoxy-L-arabinose (L-Ara4N) to the phosphate groups of lipid A of the lipopolysaccharide (LPS), a capability encoded by the arnBCADTEF-PA3559 (PA3552PA3559) operon (Ernst et al., 1999; McPhee et al., 2003; Moskowitz et al., 2004). The regulation of the operon is complex and involves at least two separate two-component regulatory systems (TCSs) encoded by the genes phoPQ and pmr $A B$, which, in response to low divalent ion concentrations in the environment, promote $\operatorname{arn} B$ transcription (McPhee et al., 2003; Moskowitz et al., 2004). Moreover, expression of the $a r n B$ operon is induced by a number of CAMPs even under high $\mathrm{Mg}^{2+}$ conditions, a response that seems to be independent of both phoPQ and pmrAB (McPhee et al., 2003). Recently, a novel TCS encoded by parRS has been implicated in the recognition of a subset of CAMPs including the polymyxins (Fernández et al., 2010), but molecular details of the mechanisms underlying CAMP induction in $P$. aeruginosa are largely un-elucidated. In this work, we investigate the CAMP recognition pathway by transposon mutagenesis and screening for mutants with changed regulation of the LPS modification operon $\operatorname{arnB}$. We have identified a novel component in the CAMP recognition pathway that is required for $P$. aeruginosa to sense the presence of CAMPs in the growth environment. Additionally, we show that this putative membrane protein is required for the formation of colistin-tolerant subpopulations in $P$. aeruginosa hydrodynamic flow chamber biofilms.

\section{METHODS}

Bacterial strains and media. The bacterial strains and plasmids used in this study are described in Supplementary Table S1 (available with the online version of this paper).

Escherichia coli and P. aeruginosa strains were routinely grown in Luria-Bertani broth, BM2-glucose minimal medium containing low $(5 \mu \mathrm{M})$ or high $(500 \mu \mathrm{M})$ concentrations of $\mathrm{MgSO}_{4}$ (Gilleland et al., 1974) and AB minimal medium (ABTG) (Clark \& Maaloe, 1967). For plasmid maintenance in $E$. coli the medium was supplemented with $35 \mu \mathrm{g}$ kanamycin $\mathrm{ml}^{-1}, 100 \mu \mathrm{g}$ ampicillin $\mathrm{ml}^{-1}, 15 \mu \mathrm{g}$ gentamicin $(\mathrm{Gm}) \mathrm{ml}^{-1}$ or $8 \mu \mathrm{g}$ chloramphenicol $\mathrm{ml}^{-1}$. For marker selection in $P$. aeruginosa $\mathrm{PAO} 1,30 \mu \mathrm{g} \mathrm{Gm} \mathrm{ml} \mathrm{g}^{-1}, 50 \mu \mathrm{g}$ tetracycline $\mathrm{ml}^{-1}$ and $200 \mu \mathrm{g}$ carbenicillin $(\mathrm{Cb}) \mathrm{ml}^{-1}$ were used, as appropriate. Colistin sulfate was used in concentrations stated in the text. All antibiotics were obtained from Sigma, unless otherwise stated.
In vitro DNA manipulation. PCR amplification was performed in a T3 Thermocycler PCR machine from Biometra using the Expand high-fidelity system (Roche) using primers presented in Supplementary Table S1. Electroporation was performed by using a Gene Pulser (Bio-Rad), as recommended by the manufacturer, with $50 \mu \mathrm{l}$ bacterial cells and 10-100 ng DNA. DNA extraction, treatment with modifying enzymes and restriction endonucleases, ligation of DNA fragments with T4 ligase, and transformation of E. coli were performed using standard methods (Ausubel et al., 1996).

Gene expression analysis. For monitoring of arnB operon expression in biofilms, a transcriptional fusion to green fluorescent protein (GFP) inserted at the $\operatorname{Tn} 7$ insertion site on the $P$. aeruginosa chromosome was constructed as described by Haagensen et al. (2007). Mini-Tn7 mobilization into the $P$. aeruginosa chromosome was performed according to the method described by Koch et al. (2001). Regulation of the $\operatorname{arnB}$ operon was investigated by growing the bacterial strains in $14 \mathrm{ml}$ Falcon plastic tubes with $1 \mathrm{ml}$ of the different media overnight at $37^{\circ} \mathrm{C}$ with shaking. Cells $\left(10^{6}\right)$ were transferred to new media and Novispirin G10 and colistin were directly added or the cells were grown to mid-exponential growth phase at $37{ }^{\circ} \mathrm{C}$ with shaking as stated in the text. For each strain and growth medium, three independent cultures were prepared. The samples were diluted in $5 \mathrm{ml}$ Falcon tubes with $0.9 \% \mathrm{NaCl}$ and the GFP fluorescence of the bacterial cells was quantified using a FACS Vantage SE flow cytometer (Becton Dickinson).

Transposon mutagenesis. Transposon insertions in the PAO1 chromosome were generated by mating strain AFP21 carrying the ParnB:: $g f p$ reporter fusion with E. coli strain SNZ15 (ISphoA/hah insertions) according to the method described by Jacobs et al. (2003), using the addition of $20 \mu \mathrm{g} \mathrm{Gm} \mathrm{ml} \mathrm{g}^{-1}$ in the selective plates for counter-selection of the E. coli donor.

Transposon insertion locations were determined by a two-stage semidegenerate PCR protocol as described by Jacobs et al. (2003). PCR fragments were purified using the NucleoSpin Extract II kit (Macherey-Nagel) according to the manufacturer's instructions. The PCR products were sequenced at GATC Biotech. The transposable element was excised from the chromosome of the transposon mutants by Cre-facilitated recombination of the loxP sites essentially as described by Manoil (2000).

Generation of unmarked $\boldsymbol{P}$. aeruginosa deletion mutants. Deletion mutants were generated as described by Choi \& Schweizer (2005). The gene disruption cassettes were transferred into $P$. aeruginosa $\mathrm{PAO} 1$ or mutants by triparental mating using the helper strain E. coli HB101/pRK600. Allelic exchange as indicated by a Gmresistant, sucrose-resistant, $\mathrm{Cb}$-sensitive phenotype was verified by PCR using the primers, gene-UpF-GWL and gene-DnR-GWR. Generation of unmarked deletion mutants was achieved by transforming electro-competent $P$. aeruginosa with $\mathrm{pFLP} 2$ as described previously (Choi \& Schweizer, 2005). Deletion of the $\mathrm{Gm}^{\mathrm{r}}$ marker was verified by colony PCR.

Construction of complementation plasmid for the PA5003 gene. The PA5003 gene was amplified from P. aeruginosa PAO1 chromosomal DNA using the primers PA5003 EcoRI and PA5003 $\mathrm{XbaI}$ (Supplementary Table S1). The PCR product was digested by using EcoRI and $\mathrm{XbaI}$ and ligated into the corresponding sites in the multiple cloning site of pUCP22 and electroporated into E. coli $\mathrm{DH} 5 \alpha$, resulting in the PA5003 complementation plasmid pUCP22-PA5003.

MIC determinations. For the bacterial strains, the MIC of colistin was determined by twofold serial dilutions of antimicrobial peptide substance in Falcon plastic tubes with $1 \mathrm{ml}$ growth medium. Each tube was inoculated with $1.0 \times 10^{6}$ c.f.u. $\mathrm{ml}^{-1}$. For each strain and 
growth medium, three independent cultures were prepared. The growth of the cultures was measured after $24 \mathrm{~h}$ at $600 \mathrm{~nm}$ with a UV1201 spectrophotometer from Shimadzu. The MIC was determined as the lowest concentration of colistin that completely inhibited bacterial growth.

Biofilm cultivation. Biofilms were grown at $30{ }^{\circ} \mathrm{C}$ in flow chambers with individual channel dimensions of $1 \times 4 \times 40 \mathrm{~mm}$. The flow system was assembled and prepared as described previously (Haagensen et al., 2007). The flow chambers were inoculated by injecting $250 \mu \mathrm{l}$ overnight culture diluted to $\mathrm{OD}_{600} 0.001$ into each flow channel with a small syringe. After inoculation, flow channels were left without flow for $1 \mathrm{~h}$, after which medium flow was started using a Watson Marlow $205 \mathrm{~S}$ peristaltic pump. Each channel was supplied with a flow of $3 \mathrm{ml} \mathrm{AB}$ medium supplemented with $0.3 \mathrm{mM}$ glucose $\mathrm{h}^{-1}$. The mean flow velocity in the flow cells was $0.2 \mathrm{~mm} \mathrm{~s}^{-1}$. The bacterial red fluorescent viability stain propidium iodide (PI) was added to biofilm medium 15 min before and during exposure to 0,8 , 16 or $25 \mu \mathrm{g}$ colistin $\mathrm{ml}^{-1}$ (Polymyxin E, Sigma). Red fluorescing and green fluorescing cells from a colistin-treated biofilm were sorted by fluorescence-activated cell sorting (FACS; Becton Dickinson FACS Vantage SE) and plated on Luria-Bertani plates to verify that red fluorescing cells were not viable and green fluorescing cells were viable.

Microscopy and image processing. All microscopic observations were completed using a Zeiss LSM510 confocal laser scanning microscope (Carl Zeiss) equipped with an argon and a NeHe laser and detectors and filter sets for simultaneous monitoring of GFP (excitation, $488 \mathrm{~nm}$; emission, $517 \mathrm{~nm}$ ) and red fluorescence emitted from PI (excitation, $543 \mathrm{~nm}$; emission, $565 \mathrm{~nm}$ ). Images were obtained using a $63 \times / 1.4$ Plan-APOChromat differential interference contrast objective or a $40 \times / 1.3$ Plan-Neofluar oil objective. Multichannel simulated fluorescence vertical cross sections images were generated using the IMARIS software package (Bitplane).

\section{RESULTS}

\section{Antimicrobial peptides can induce the LPS modification operon independently of phoPQ and pmrAB}

The $P$. aeruginosa arnBCADTEF-PA3559 operon (arnBoperon) consists of eight genes which are implicated in the synthesis and processing of $\mathrm{L}-\mathrm{Ara} 4 \mathrm{~N}$ and linking it to the phosphate groups of lipid A, leading to increased polymyxin $\mathrm{B}$ tolerance and the formation of colistin-tolerant subpopulations in flow chamber biofilms (Ernst et al., 1999; Haagensen et al., 2007; McPhee et al., 2003; Moskowitz et al., 2004). Expression of this operon is induced by the CAMPs themselves, if present in the growth environment (McPhee et al., 2003). In order to investigate antimicrobial peptide recognition and the regulation of the $\operatorname{arnB}$ operon, we constructed a GFP transcriptional reporter fusion to the $\operatorname{arn} B$ promoter and incorporated it into the $P$. aeruginosa PAO1 chromosome using a mini-Tn7-based method (Haagensen et al., 2007; Lambertsen et al., 2004). We chose GFP fusions because they are extensively used as transcriptional reporters to monitor gene expression in bacteria and allow for the direct use in downstream applications such as FACS and in investigations involving confocal laser scanning microscopy (CLSM) (Bollenbach et al., 2009; Christensen et al., 1999; Southward \& Surette, 2002; Winfield \&
Groisman, 2004; Zaslaver et al., 2006). Since the direct influence of $p m r A B$ and $p h o P Q$ on CAMP recognition has been unclear (McPhee et al., 2003), we investigated the ability of $P$. aeruginosa to recognize two structurally distinct CAMPs: the linear peptide Novispirin G10 and the cyclic lipopeptide colistin in PAO1 wild-type and phoPQ and pmrAB mutant backgrounds. We also constructed a pmrABphoPQ deletion double mutant to ensure that any effect of the TCSs on CAMP recognition would not be masked by the presence of multiple mechanisms of recognition involving both $\mathrm{PhoP}-\mathrm{PhoQ}$ and $\mathrm{PmrA}-\mathrm{PmrB}$. We verified the function of the reporter fusion by investigating the GFP fluorescence of PAO1 and the deletion mutants grown in BM2 medium with $500 \mu \mathrm{M} \mathrm{Mg}^{2+}$ (BM2high) and $5 \mu \mathrm{M} \mathrm{Mg}^{2+}$ (BM2-low). Low $\mathrm{Mg}^{2+}$ induced the reporter tenfold in PAO1 wild-type (wt) while no increased fluorescence could be detected in the double mutant, which is in agreement with previous work using other reporter systems (Supplementary Fig. S1, available with the online version of this paper) (McPhee et al., 2003). Addition of subminimal inhibitory concentrations (subMIC) $(4 \mu \mathrm{g}$ $\mathrm{ml}^{-1}$ ) of Novispirin G10 in BM2-high medium induced the reporter 13-fold in PAO1 wt and was induced to a greater extent in the phoPQ and $p m r A B$ deletion mutants and the double mutant (Fig. 1 and Supplementary Table S2). Similarly, deletion of $p h o P Q$ and $p m r A B$ did not affect the sensing of colistin in ABTG minimal medium as addition of subMIC $\left(0.5 \mu \mathrm{g} \mathrm{ml}^{-1}\right)$ colistin induced the ParnB::gfp reporter by two- to threefold in all strains (Fig. 1 and Supplementary Table S2). The ABTG medium contained $1000 \mu \mathrm{M} \mathrm{Mg}^{2+}$ and an additional $100 \mu \mathrm{M} \mathrm{Ca}^{2+}$ which reduce the basal $\operatorname{arn} B$ expression of wt PAO1 to the levels comparable to those observed in the phoPQpmrAB double mutant in BM2 medium (Supplementary Fig. S1 and Supplementary Table S3). Our data show that $P$. aeruginosa recognizes antimicrobial peptides through a molecular pathway independent of both $p h o P Q$ and $p m r A B$. However, the absolute levels of expression are influenced by both TCSs.

\section{Identification of additional genes involved in LPS modification operon regulation by transposon mutagenesis}

To identify the molecular factors underlying antimicrobial peptide recognition we performed a whole-genome transposon insertion mutagenesis of a PAO1 strain carrying the ParnB:: $g p$ reporter (AFP21), using the Tn5 IS50L derivative, ISphoA/hah, conferring tetracycline resistance (Bailey \& Manoil, 2002). The mutagenesis was followed by a screen on both BM2-high and BM2-low media plates searching for colonies with altered GFP fluorescence, i.e. colonies that were either brighter or darker compared with un-mutagenized AFP21. More than 30000 colonies were visually inspected and approximately 300 candidate colonies were selected for further characterization. By FACS analysis we were able to confirm that the ParnB::gfp reporter was differentially expressed in 43 of the 300 tetracycline resistant mutants and the mapping of the transposon insertions indicates 

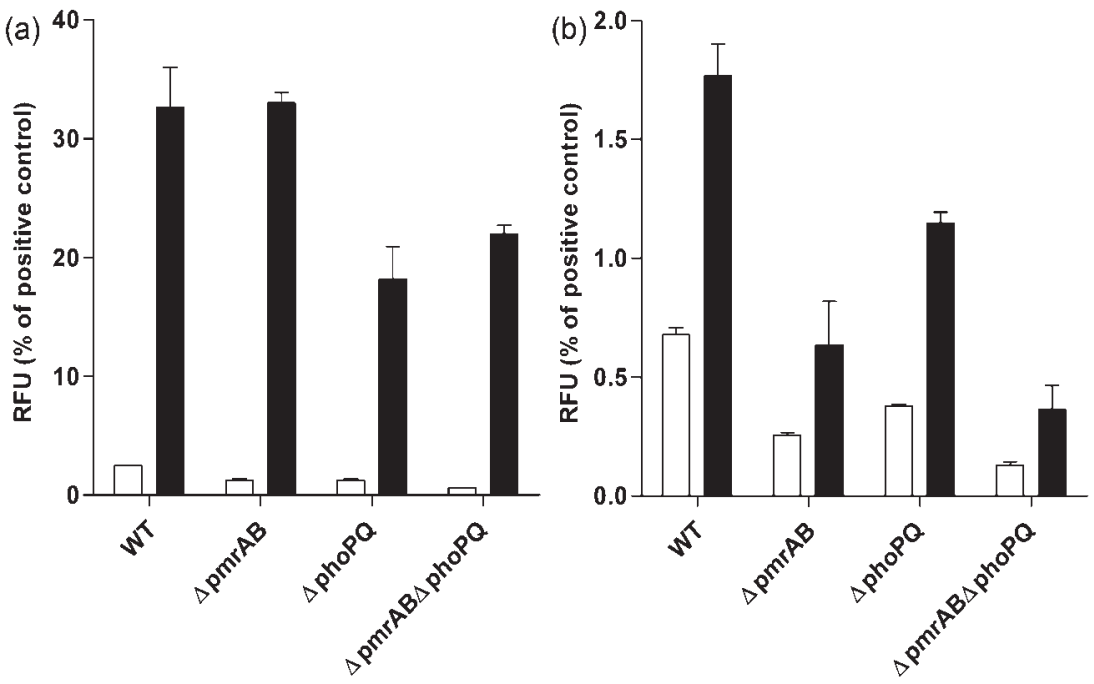

Fig. 1. CAMPs can induce arnB expression independently of the PhoP-PhoQ and PmrAPmrB systems. Expression of arnB in cells grown to mid-exponential phase. (a) Bars indicate BM2-high medium without (open) or with (closed) $4 \mu \mathrm{g}$ Novispirin G10 ml$~^{-1}$. (b) Bars indicate ABTG medium without (open) or with (closed) $0.5 \mu \mathrm{g}$ colistin $\mathrm{ml}^{-1}$. Results shown are the mean \pm SD of three experiments. Values are given as the percentage fluorescence compared with the positive control (PAO1 attTn7-P $\mathrm{A} 1 / 04 / 03:: g^{\text {mpt }^{\text {mut }}}{ }^{*}$ ).

that insertion in 29 genes affects $\operatorname{arnB}$ expression (Table 1). The major target for transposon insertion was the oprHphoPphoQ (PA1178-PA1180) operon since 13 of the 43 independent mutants had insertions in this locus. All insertions in the operon led to upregulation of ParnB::gfp fluorescence both on BM2-high plates and in liquid culture in the same media (Table 1 and Supplementary Table S4). Moreover, a large number of mutants had transposon insertions in genes encoding putative (10/43) outer membrane RND transporters and other transport proteins (Table 1), indicating that the transport into and out of the cell affects the regulation of the $\operatorname{arnB}$ promoter. Our results also show that proteins encoded by the $\operatorname{arnB}$ operon itself affect the expression of the operon as transposon mutant L15 carrying an insertion in the arnA gene had increased expression of the ParnB::gfp reporter relative to PAO1 wt (Table 1). Interestingly, we isolated mutants with transposon insertions in the PA2798-PA2797 operon that were more resistant towards colistin despite lower levels of arnB expression than PAO1 wt (Table 1, Supplementary Fig. S2), indicating that additional factors may contribute to CAMP tolerance in $P$. aeruginosa.

\section{The PA5003 gene is necessary for CAMP recognition}

To identify insertion mutants that were perturbed in their CAMP recognition, the transposon mutants were screened for loss of CAMP-mediated induction of the $\operatorname{arnB}$ operon by using a microtitre-based assay.

One mutant, designated H116, had increased GFP fluorescence on plates but not in BM2 or ABTG media and was confirmed by FACS analysis to be unable to induce the $a r n B$ promoter when Novispirin G10 or colistin was added to the media (Fig. 2a, b) (Newman-Keuls test, all $P>0.05)$. The mutant had an intact response to changes in $\mathrm{Mg}^{2+}$ concentration (Fig. 2c). The transposon was mapped to the PA5004 gene, which is part of a predicted operon from PA5005 to PA5001. Excision of the transposon led to recovered $a r n B$ induction in response to the addition of both CAMPs, indicating that the insertion affects genes downstream of PA5004 (Fig. 4a and b) (Newman-Keuls test, all $P<0.05)$.

Disruption of PA5002 did not change CAMP recognition (Fig. 2a, b), indicating that the lack of CAMP induction in mutant H116 was due to polar effects of the transposon insertion on PA5003. However, we failed to generate a PA5003 deletion mutant, implying that the PA5003 gene may be essential for viability in $P$. aeruginosa under the conditions tested. To test the requirement of PA5003 for CAMP activation, the gene was cloned into the high copy number plasmid pUCP22 and transformed into transposon mutant H116. Subsequent FACS analysis showed that PA5003 in trans is sufficient to restore CAMP-mediated $\operatorname{arnB}$ induction in the mutant (Fig. $3 \mathrm{a}$ and b) for both Novispirin G10 and colistin.

\section{PA5003 is essential for tolerant subpopulation formation in $\boldsymbol{P}$. aeruginosa biofilms}

We have previously shown that arnB operon expression and LPS modification is correlated with the formation of a colistin-tolerant subpopulation when $P$. aeruginosa is grown in hydrodynamic flow chamber biofilm systems (Haagensen et al., 2007). To investigate how PA5003 affects colistin tolerance in $P$. aeruginosa biofilms, we established flow chamber biofilms seeded with the strains harbouring the $\mathrm{P} a r n B:: g f p$ reporter construct and incubated for 3 days at $30{ }^{\circ} \mathrm{C}$ in ABTG medium. The biofilms were then challenged with three different concentrations (8, 16 and $25 \mu \mathrm{g} \mathrm{ml}^{-1}$ ) of colistin for $24 \mathrm{~h}$ and then stained with PI to distinguish live, GFP-expressing cells and dead PI-stained cells. As expected, the untreated control biofilm exhibited only a few dead (red) cells spread throughout the biofilm and very low to undetectable levels of GFP fluorescence due to little $\operatorname{arnB}$ promoter activity (Fig. 4a). To establish that 
Table 1. Overview of transposon mutants and confirmed phenotypes

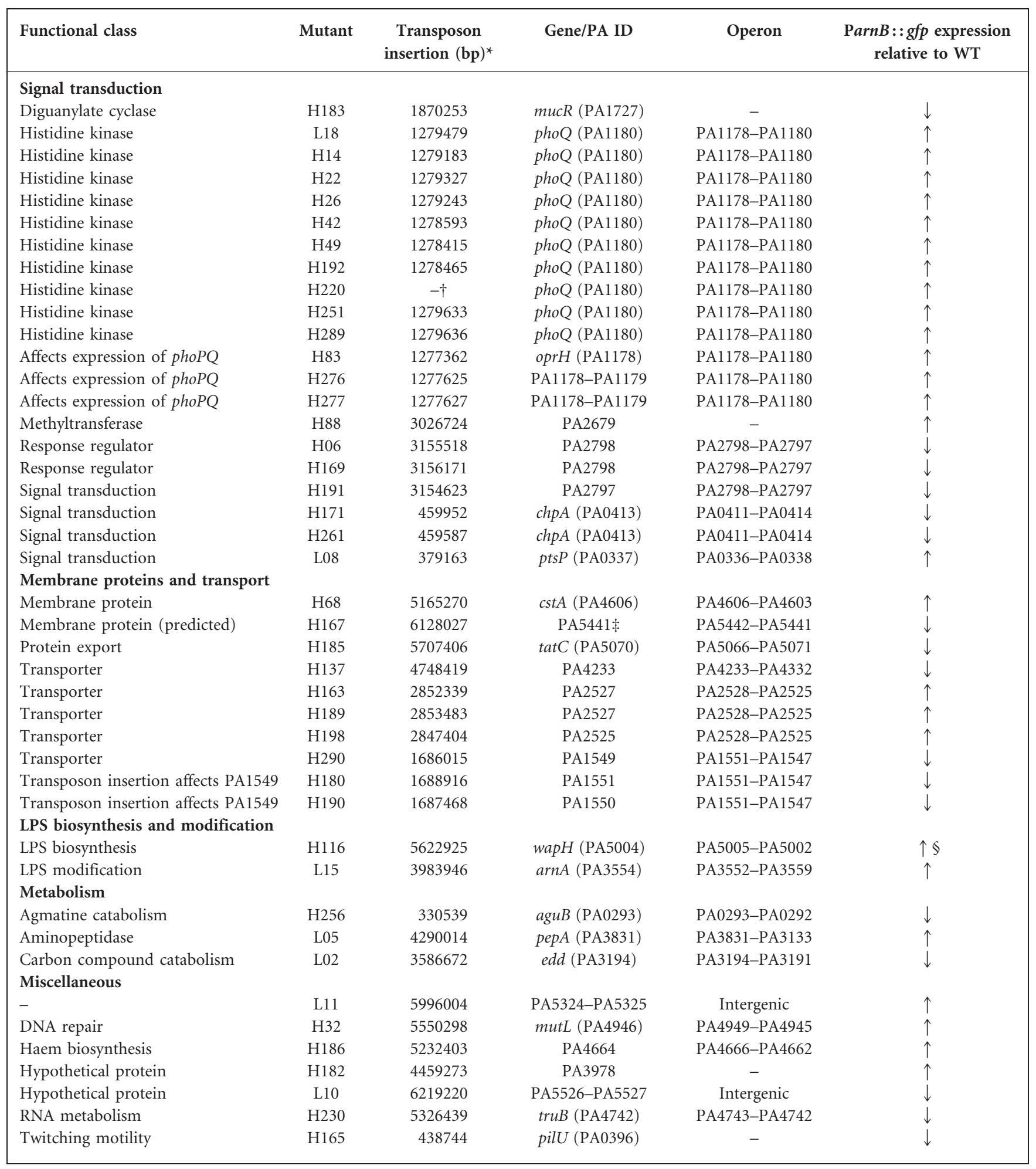

${ }^{*}$ Transposon insertion sites (bp) in the PAO1 chromosome (complete genome, GenBank accession no. AE004091.2).

$\dagger$ Transposon insertion in $p h o Q$ confirmed by PCR.

$\$$ Transposon insertion 30 bp upstream PA5441 coding sequence.

§CAMP did not induce ParnB::gfp in liquid culture. 

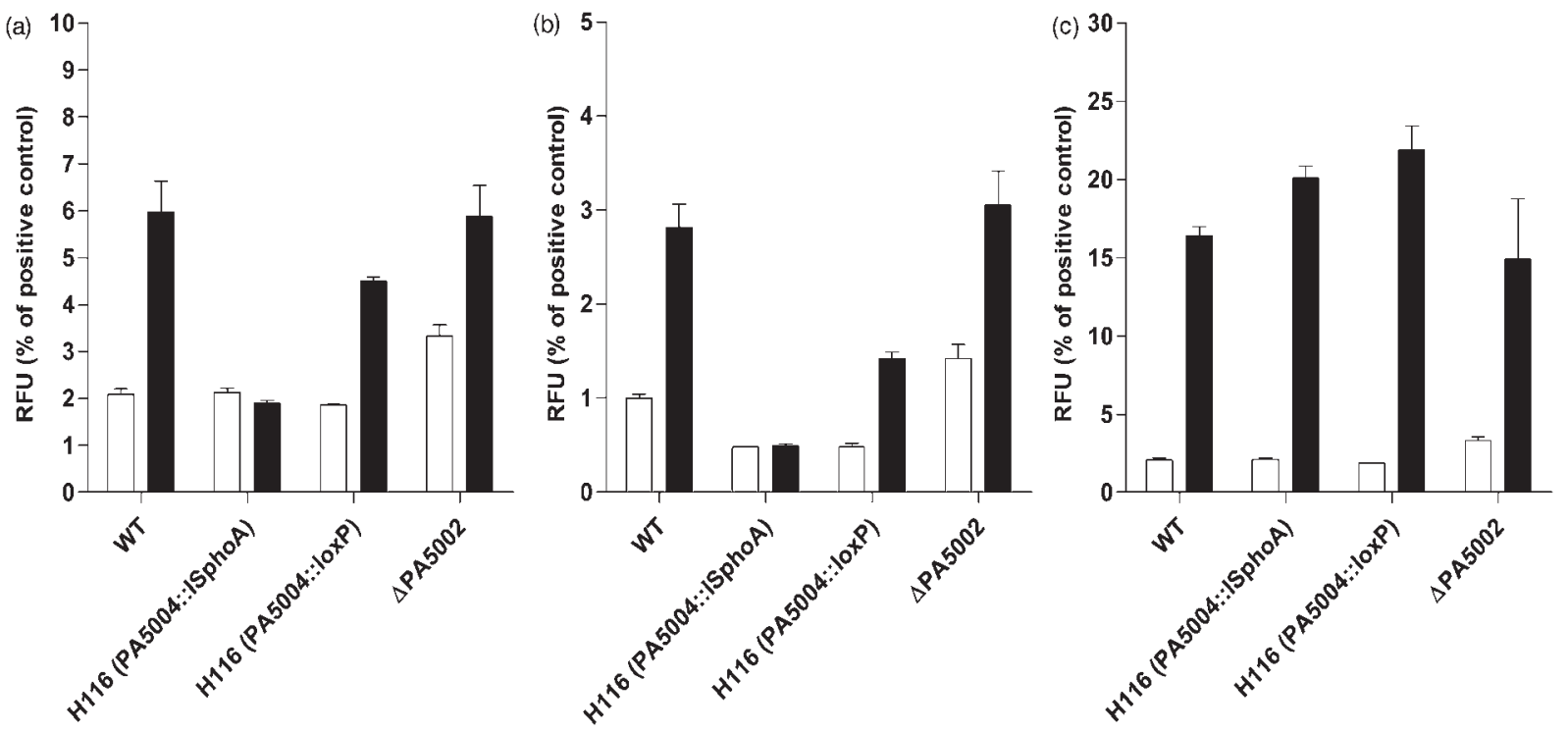

Fig. 2. Induction and regulation of ParnB::gfp expression in mutants with gene disruption in the PA5005-PA5001 operon. arnB expression in stationary phase cells is shown. (a) Bars indicate BM2-high $\mathrm{Mg}^{2+}$ medium without (open) and with (closed) $2 \mu \mathrm{g}$ Novispirin $\mathrm{G} 10 \mathrm{ml}^{-1}$. (b) Bars indicate ABTG medium without (open) and with (closed) $0.5 \mu \mathrm{g} \mathrm{colistin} \mathrm{ml}^{-1}$. (c) Bars indicate BM2-high $\mathrm{Mg}^{2+}$ (open) and BM2-low $\mathrm{Mg}^{2+}$ (closed) media. Results shown are the mean $\pm \mathrm{SD}$ of three experiments. Values are given as the percentage fluorescence compared with the positive control (PAO1 attTn7-P $\left.\mathrm{A} 1 / 04 / 03:: g^{\mathrm{mut} 3^{*}}\right)$.

there were actually living cells in the untreated flow chambers, the green fluorescent live cell stain Syto 9 was added to the controls showing an extensive, structured biofilm (Fig. 4a, insets). When challenged with $16 \mu \mathrm{g}$ colistin $\mathrm{ml}^{-1}$, the biofilm formed by the wt PAO1 strain exhibited an extensive killing of bacterial cells at the inside of the biofilm closest to the glass surface (Fig. 4b). However, as expected, a significant proportion of the cells in the biofilm survived the challenge, forming a green fluorescent arnB-expressing subpopulation, covering the dead cells (Fig. 4b). The surviving cells also continued to grow if colistin treatment was continued (data not shown; Haagensen et al., 2007). In the H116 biofilm challenged with the same colistin concentration, no arnB expression or surviving cells could be detected and no growth in the biofilm could be detected after an additional $24 \mathrm{~h}$ incubation with colistin (Fig. 4c). To ensure that there were no surviving cells that were not expressing $\operatorname{arnB}$ in significant amounts, the treated H116 mutant biofilm was stained with the green fluorescent viability stain Syto 9 but no living cells were detected (data not shown). In biofilms formed by $\mathrm{H} 116$ with PA5003 on a multi-copy plasmid, an extensive subpopulation of surviving, arnBexpressing cells could be detected (Fig. 4d). The surviving PA5003-complemented cells had a similar spatial localization, on top of and covering the dead cells, to the wild-type. Comparable results were obtained for biofilms of the different strains at $8 \mu \mathrm{g} \mathrm{ml}^{-1}$ and
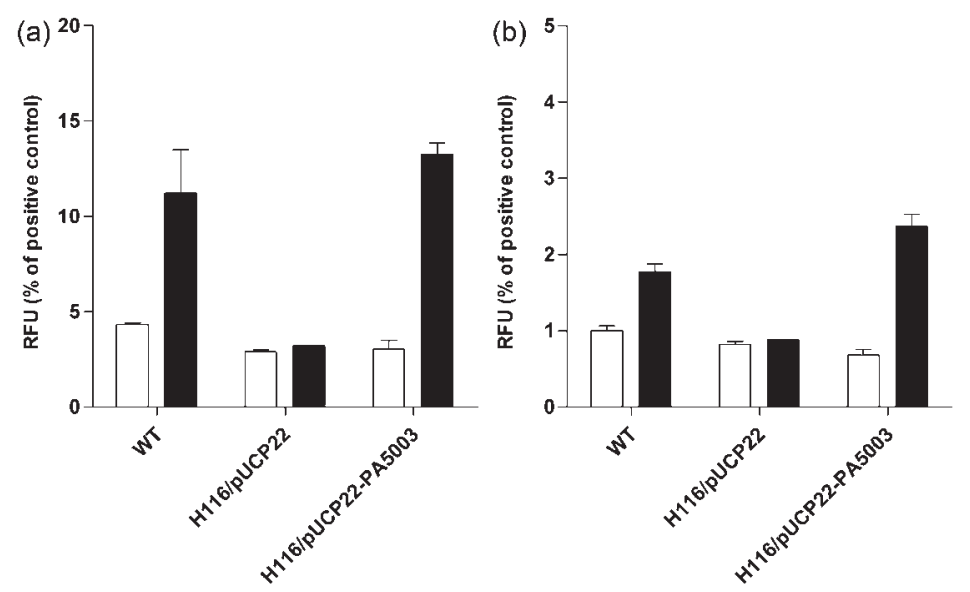

Fig. 3. The PA5003 gene encoding a Mig-14like protein is required for CAMP-mediated induction of ParnB::gfp. (a) Bars indicate $\mathrm{BM} 2-$ high $\mathrm{Mg}^{2+}$ medium without (open) and with (closed) $2 \mu \mathrm{g}$ Novispirin $\mathrm{G} 10 \mathrm{ml}^{-1}$. (b) Bars indicate ABTG medium without (open) and with (closed) $0.5 \mu \mathrm{g}$ colistin $\mathrm{ml}^{-1}$. Results shown are the mean \pm SD of three experiments. Values are given as the percentage fluorescence compared with the positive control (PAO1 attTn7-P $\mathrm{A} 1 / 04 / 03::$ gfp $^{\text {mut3 }}{ }^{*}$ ). 

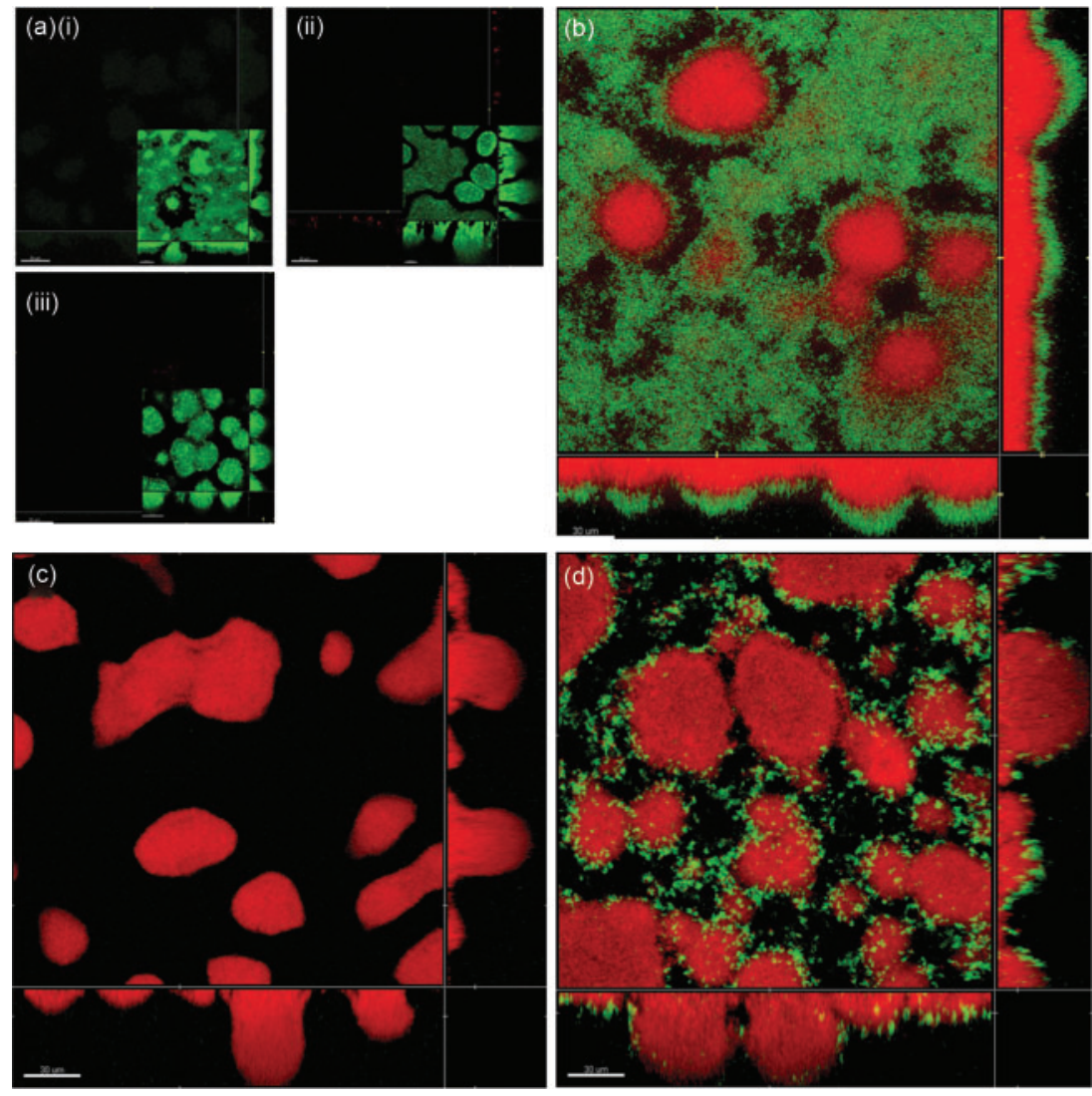

Fig. 4. PA5003 is required for colistin-tolerant subpopulation formation in flow chamber biofilms. (a) Untreated wild-type PAO1 (i), untreated $\mathrm{H} 116$ mutant (ii) and untreated H116/pUCP22-PA5003 (iii). The insets depict untreated biofilms stained with the green live stain Syto 9 to show the extent of biofilm biomass. Green represents arnBexpressing cells while red represents dead cells due to staining by the dead indicator PI. (b-d) CLSM micrographs showing the distribution of ParnB::gfp-expressing cells and dead cells in 4-day-old $P$. aeruginosa biofilms after $24 \mathrm{~h}$ challenge with $16 \mu \mathrm{g}$ colistin $\mathrm{ml}^{-1}$ of wild-type PAO1 (b), H116 (c) and $\mathrm{H} 116$ / pUCP22-PA5003 (d). The micrographs show a horizontal section with two flanking images representing sections in the $x z$ and $y z$ planes, respectively. Bars, $30 \mu \mathrm{m}$.
$25 \mu \mathrm{g} \mathrm{ml}^{-1}$ (Supplementary Fig. S3). These results indicate that expression of the PA5003 gene is necessary for the formation of colistin-tolerant subpopulations in $P$. aeruginosa biofilms and that peptide recognition is essential for this process. The effect of Novispirin G10 on biofilm-growing $P$. aeruginosa was also investigated by challenging 2-day-old flow chamber biofilms with $20 \mu \mathrm{g}$ Novispirin $\mathrm{G} 10 \mathrm{ml}^{-1}$. A tolerant arnB-expressing subpopulation was formed in the PAO1 wt biofilm while no such population was evident in the H116 mutant (Fig. $5 \mathrm{a}, \mathrm{b})$. In biofilms formed by H116 with PA5003 on a multi-copy plasmid, a subpopulation of surviving arnBexpressing cells could be detected with the same spatial distribution of arnB-expressing cells as the wild-type (Fig. 5c). These data indicate that even though Novispirin G10 and colistin have very different structures, these peptides are recognized by $P$. aeruginosa through similar pathways.

\section{DISCUSSION}

The recognition of and response to CAMPs are important for successful colonization and survival of micro-organisms interacting with eukaryotic hosts. In Gram-negative bacteria, the enzymes encoded by the $\operatorname{arn} B$ operon are essential for the ability to survive in the presence of CAMPs such as polymxin B and colistin and also in the presence of the different classes of host-derived antimicrobial peptides
(Breazeale et al., 2002, 2003; Gunn et al., 1998; McPhee et al., 2003; Trent et al., 2001). In S. enterica, the ability to modify the outer membrane lipid A molecules through the action of the Arn proteins is essential for full virulence (Gunn, 2001). Moreover, in organisms such as E. coli and $P$. aeruginosa this operon is involved in the formation of CAMP-tolerant subpopulations in biofilms (Folkesson et al., 2008; Haagensen et al., 2007).

The regulation of the arnB operon in P. aeruginosa is highly complex reflecting the diverse roles of $\mathrm{N}_{4}$-aminoarabinose lipid A modification in the normal function of the outer membrane. The two TCSs PhoP-PhoQ and PmrA-PmrB synergistically control arnB promoter activity in response to the levels of divalent cations, primarily $\mathrm{Mg}^{2+}$, in the environment (Supplementary Fig. S1) (McPhee et al., 2003). In S. enterica, the PhoP-PhoQ system seems to be responsible for the recognition of CAMPs and for the induction of the $\operatorname{arnB}$ operon leading to increased peptide tolerance (Bader et al., 2005). However, our pmrABphoPQ double mutant studies show that peptide-mediated induction of the $\operatorname{arn} B$ operon in $P$. aeruginosa can occur independently of both phoPQ and pmrAB (Fig. 1 and Supplementary Table S2). This implies that there are additional factors involved in the process of CAMP sensing in $P$. aeruginosa. In the transposon mutagenesis presented here, we screened more than 30000 mutants for changed arnB expression, i.e. mutants with either increased or decreased fluorescence. This was possible because the 

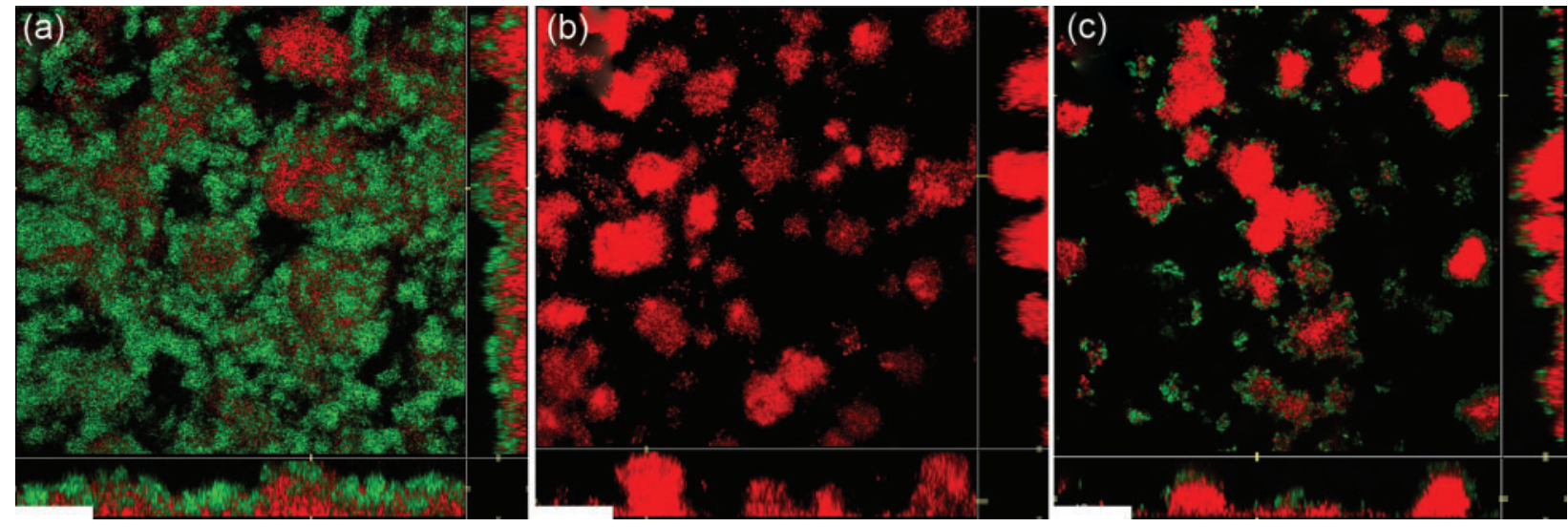

Fig. 5. PA5003 is required for Novispirin G10-tolerant subpopulation formation in flow chamber biofilms. CLSM micrographs showing the distribution of ParnB : : gfp-expressing cells and dead cells in 2-day-old $P$. aeruginosa biofilms after $24 \mathrm{~h}$ challenge with $20 \mu \mathrm{g}$ Novispirin $\mathrm{G} 10 \mathrm{ml}^{-1}$ of wild-type PAO1 (a), H116 (b) and H116/pUCP22-PA5003 (c). Green represents arnBexpressing cells while red represents dead cells due to staining by the dead indicator PI. The micrographs show a horizontal section with two flanking images representing sections in the $x z$ and $y z$ planes, respectively. Bars (lower left corner), $40 \mu \mathrm{m}$.

promoter is not completely repressed on the BM2 medium plates. The majority of the isolated mutants were upregulated in $\operatorname{arn} B$ expression (Table 1). This may be due to the fact that the screen primarily identified putative repressors of the $a r n B$ operon since the transposon most likely causes loss of function mutations in the genes into which it inserts. This is demonstrated by the many insertions in $p h o Q$ and the oprHphoPQ operon (Table 1). Conceivably, it is easier to identify colonies by visual inspection, which are brighter than background fluorescence compared with those with dimmer fluorescence; this could be an important factor as the majority of the mutants were isolated from BM2-high plates. It is also possible that insertions severely affecting the normal function of the $\operatorname{arnB}$ system will have an effect on the viability of the bacteria, especially on the BM2-low plates.

Transposon mutagenesis revealed that the PA5003 gene is required for CAMP-mediated induction of the $\operatorname{arn} B$ operon. The putative protein encoded by PA5003 in the $P$. aeruginosa $\mathrm{PAO} 1$ chromosome has $45 \%$ similarity to the Mig-14 protein of $S$. enterica serovar Typhimurium (Winsor et al., 2009). This protein is required for full virulence and is under the control of the Salmonella PhoPPhoQ system (Valdivia et al., 2000). The mig-14 gene is strongly induced by polymyxin $\mathrm{B}$ in a phoP-independent manner and is necessary for resistance towards polymyxin B and other CAMPs (Brodsky et al., 2002, 2005). The $P$. aeruginosa PA5003 is located in the core oligosaccharide biosynthesis gene cluster, downstream of PA5004 encoding a putative glycosyltransferase WapH (Lam et al., 2004; Matewish, 2004). Repeated attempts in our laboratory and in other laboratories (Lam et al., 2004) to create a null mutation in PA5003 have failed, indicating that the gene product of the ORF is essential for $P$. aeruginosa viability. These results are in contrast with those obtained for $S$. enterica, where deletion mutants have been isolated (Brodsky et al., 2002).

It is possible that the insertion in PA5004 in mutant $\mathrm{H} 116$ allows a low level expression of the PA5003 gene, presumably through an alternative promoter in the reading frame of PA5004, which implies that proper regulation of PA5003 expression is required for CAMP-mediated induction of the $\operatorname{arn} B$ operon. Experiments to resolve this problem are ongoing in the laboratory.

The exact mechanism by which mig-14 contributes to Salmonella pathogenicity is not known although it has been shown to be associated with the inner membrane and to reduce the binding of CAMPs to the bacterial cell (Brodsky et al., 2005). Even less is known about the biological role of the protein encoded by PA5003 and its function in oligosaccharide biosynthesis is not well elucidated (King et al., 2009; Lam et al., 2004). Recently, Fernández et al. (2010) identified a novel TCS, ParR-ParS, which is required for the induction of the $\operatorname{arnB}$ promoter upon exposure to certain CAMPs. Interestingly, mutation of parRS only affected the sensing of polymyxins and the tryptophan-rich peptide indolicidin, indicating that there are specific recognition pathways for specific structural subclasses of antimicrobial peptides or that ParR-ParS indirectly affects the regulation of the $\operatorname{arn} B$ system. Disruption of parRS downregulates the expression of $p m r A B$, which can have unpredictable effects on the basal level of $a r n B$ expression, since complete removal of $p m r A B$ significantly decreases this expression (Fig. 2). It is important to clarify if the products of PA5003 and parRS are parts of the same regulatory network or if they act independently on $\operatorname{arnB}$ expression and CAMP recognition. Recent studies may imply an association between LPS structure, biosynthesis and CAMP recognition. Srinivas 
et al. (2010) recently found that peptidomimetics based on the antimicrobial peptide protegrin I target the assembly of the LPS molecules in the outer leaflet on the outer membrane in $P$. aeruginosa. Furthermore, lantibiotics and the fungal defensin derivative plectasin target the cell wall precursor lipid II in Mirococcus flavus and Bacillus subtilis, respectively (Breukink et al., 1999; Brötz et al., 1998; Schneider et al., 2010). It is possible that important cellular processes such as cell wall synthesis and LPS assembly may be general targets for antimicrobial peptides, including colistin and Novispirin G10. Given that PA5003 is situated in a core oligosaccharide biosynthesis gene cluster it is possible that the normal function of the protein product of PA5003 is to monitor the biosynthesis or assembly of LPS. Impairment of LPS assembly or perturbation of outer membrane integrity due to the presence of CAMPs could act as a signal to the PA5003-encoded protein and initiate an uncharacterized signalling cascade inducing the expression of many genes, including the $\operatorname{arn} B$ operon. However, it is also possible that proper regulation of PA5003 expression is required for normal membrane physiology and for the components of the recognition pathway to assemble correctly. Our results suggest that aminoarabinose lipid A modification plays a more fundamental role in membrane physiology than previously recognized. The large number of different loci affecting arnB expression, identified by the transposon mutagenesis, further supports this conclusion (Table 1). Interestingly, we have isolated a mutant with a transposon insertion in the $\operatorname{arn} B$ operon itself, which activates the expression of the reporter construct (Table 1). Apparently there is a mechanism which can monitor the level of aminoarabinose lipid A modification or the physiological consequences of the modifications on outer membrane structure, increasing the expression of the operon as a response. However, the modifications are not part of the CAMP recognition pathway because the $\operatorname{arn} B$ operon insertion mutant responds like the wild-type to CAMP challenge (Table 1).

Novispirin G10 and colistin are differentially effective in activating $\operatorname{arn} B$ operon expression. Small structural differences have been shown to have significant changes in induction levels of the operon (McPhee et al., 2003). However, the structural and biochemical mechanisms underlying these differences are not known but are probably dependent on how the peptides insert into the membrane and affect its integrity.

Biofilm formation has often been associated with a reduced susceptibility to antimicrobial challenge (Ceri et al., 1999; Nickel et al., 1985). One major mechanism behind the apparent increased resistance is subpopulation differentiation within the biofilm (Folkesson et al., 2008; Haagensen et al., 2007; Pamp et al., 2008). Many factors contribute to the formation of these populations, which are antibiotic specific and conditionally dependent on the biofilm structure, growth medium and growth state. The arnB operon is essential for the formation of CAMP-tolerant subpopulations in $P$. aeruginosa hydrodynamic flow chamber biofilms when the biofilm is challenged with colistin, but the ability to respond to the peptide seems to be tightly regulated because a large part of the biofilm population is highly susceptible to the peptide (Haagensen et al., 2007). The mechanisms regulating this differentiation are not well elucidated but recently it has been proposed that the growth state may be important to the segregation into tolerant and non-tolerant cell populations (Pamp et al., 2008). In the present work, we show that recognition of CAMPs such as colistin is a prerequisite for the induction of $\operatorname{arn} B$ expression in the tolerant subpopulation and that induction of $\operatorname{arnB}$ in the surviving population is due to a specific mechanism and is not part of a general stress response towards the action of the antibiotics (Fig. 4). The multi-copy plasmid carrying PA5003 does not completely rescue the H116 mutant from the action of the peptides. This is probably due to three interacting factors. Firstly, H116 still lacks the PA5004 gene due to the transposon insertion and it cannot be ruled out that this gene can influence the susceptibility to cationic peptides in the biofilm system, even though the MIC is not significantly different between the mutants and wt PAO1. Secondly, the PA5003 construct does not contain the native promoter and expression is driven by a constitutive promoter on the plasmid which could mean that the levels of PA5003 protein might not be optimal under all growth conditions encountered in the biofilm. Thirdly, there are most probably copy number differences and plasmid stability issues that could further augment the PA5003 expression differences between different cells within the biofilm. These factors can possibly synergistically interact to prevent $100 \%$ recovery of the arnB induction phenotype.

These results show that peptide recognition may be a process contributing to the difficulty to treat $P$. aeruginosa infections (Prince, 2002). The findings suggest that interference of the peptide recognition pathway or engineering of peptides that are less efficient in activating the $\operatorname{arn} B$ tolerance mechanism may be a way to enhance the efficacy of antimicrobial peptides in the treatment of $P$. aeruginosa biofilm infections.

\section{ACKNOWLEDGEMENTS}

This work was supported by the Danish Research Council. We would like to thank Cecilia Thomas and Rune Lyngklip Jensen for technical assistance. We would also like to thank Hans Henrik Christensen and Novozymes A/S for the kind gift of Novispirin G10.

\section{REFERENCES}

Ausubel, F. M., Brent, M. R., Kingston, R. E., Moore, D. D., Siedman, J. G., Smith, J. A. \& Struhl, K. (1996). Current Protocols in Molecular Biology. New York: Wiley.

Bader, M. W., Sanowar, S., Daley, M. E., Schneider, A. R., Cho, U., Xu, W., Klevit, R. E., Le Moual, H. \& Miller, S. I. (2005). Recognition of antimicrobial peptides by a bacterial sensor kinase. Cell 122, 461-472. 
Bailey, J. \& Manoil, C. (2002). Genome-wide internal tagging of bacterial exported proteins. Nat Biotechnol 20, 839-842.

Bollenbach, T., Quan, S., Chait, R. \& Kishony, R. (2009). Nonoptimal microbial response to antibiotics underlies suppressive drug interactions. Cell 139, 707-718.

Breazeale, S. D., Ribeiro, A. A. \& Raetz, C. R. (2002). Oxidative decarboxylation of UDP-glucuronic acid in extracts of polymyxinresistant Escherichia coli. Origin of lipid A species modified with 4amino-4-deoxy-L-arabinose. J Biol Chem 277, 2886-2896.

Breazeale, S. D., Ribeiro, A. A. \& Raetz, C. R. (2003). Origin of lipid A species modified with 4-amino-4-deoxy-L-arabinose in polymyxinresistant mutants of Escherichia coli. An aminotransferase (ArnB) that generates UDP-4-deoxy-L-arabinose. J Biol Chem 278, 24731-24739.

Breukink, E., Wiedemann, I., van Kraaij, C., Kuipers, O. P., Sahl, H. \& de Kruijff, B. (1999). Use of the cell wall precursor lipid II by a poreforming peptide antibiotic. Science 286, 2361-2364.

Brodsky, I. E., Ernst, R. K., Miller, S. I. \& Falkow, S. (2002). mig-14 is a Salmonella gene that plays a role in bacterial resistance to antimicrobial peptides. J Bacteriol 184, 3203-3213.

Brodsky, I. E., Ghori, N., Falkow, S. \& Monack, D. (2005). Mig-14 is an inner membrane-associated protein that promotes Salmonella typhimurium resistance to CRAMP, survival within activated macrophages and persistent infection. Mol Microbiol 55, 954-972.

Brogden, K. A. (2005). Antimicrobial peptides: pore formers or metabolic inhibitors in bacteria? Nat Rev Microbiol 3, 238-250.

Brötz, H., Bierbaum, G., Leopold, K., Reynolds, P. E. \& Sahl, H. G. (1998). The lantibiotic mersacidin inhibits peptidoglycan synthesis by targeting lipid II. Antimicrob Agents Chemother 42, 154-160.

Ceri, H., Olson, M. E., Stremick, C., Read, R. R., Morck, D. \& Buret, A. (1999). The Calgary Biofilm Device: new technology for rapid determination of antibiotic susceptibilities of bacterial biofilms. J Clin Microbiol 37, 1771-1776.

Choi, K. H. \& Schweizer, H. P. (2005). An improved method for rapid generation of unmarked Pseudomonas aeruginosa deletion mutants. BMC Microbiol 5, 30 .

Christensen, B. B., Sternberg, C., Andersen, J. B., Palmer, R. J., Jr, Nielsen, A. T., Givskov, M. \& Molin, S. (1999). Molecular tools for study of biofilm physiology. Methods Enzymol 310, 20-42.

Clark, D. J. \& Maaloe, O. (1967). DNA replication and division cycle in Escherichia coli. J Mol Biol 23, 99-112.

Conly, J. \& Johnston, B. (2006). Colistin: the phoenix arises. Can J Infect Dis Med Microbiol 17, 267-269.

Conrad, R. S. \& Galanos, C. (1989). Fatty acid alterations and polymyxin B binding by lipopolysaccharides from Pseudomonas aeruginosa adapted to polymyxin B resistance. Antimicrob Agents Chemother 33, 1724-1728.

Ernst, R. K., Yi, E. C., Guo, L., Lim, K. B., Burns, J. L., Hackett, M. \& Miller, S. I. (1999). Specific lipopolysaccharide found in cystic fibrosis airway Pseudomonas aeruginosa. Science 286, 1561-1565.

Fernández, L., Gooderham, W. J., Bains, M., McPhee, J. B., Wiegand, I. \& Hancock, R. E. (2010). Adaptive resistance to the "last hope" antibiotics polymyxin B and colistin in Pseudomonas aeruginosa is mediated by the novel two-component regulatory system ParR-ParS. Antimicrob Agents Chemother 54, 3372-3382.

Folkesson, A., Haagensen, J. A., Zampaloni, C., Sternberg, C. \& Molin, S. (2008). Biofilm induced tolerance towards antimicrobial peptides. PLoS ONE 3, e1891.

Frederiksen, B., Koch, C. \& Høiby, N. (1997). Antibiotic treatment of initial colonization with Pseudomonas aeruginosa postpones chronic infection and prevents deterioration of pulmonary function in cystic fibrosis. Pediatr Pulmonol 23, 330-335.
Gilleland, H. E., Jr, Stinnett, J. D. \& Eagon, R. G. (1974). Ultrastructural and chemical alteration of the cell envelope of Pseudomonas aeruginosa, associated with resistance to ethylenediaminetetraacetate resulting from growth in a $\mathrm{Mg}^{2+}$-deficient medium. J Bacteriol 117, 302-311.

Gunn, J. S. (2001). Bacterial modification of LPS and resistance to antimicrobial peptides. J Endotoxin Res 7, 57-62.

Gunn, J. S., Lim, K. B., Krueger, J., Kim, K., Guo, L., Hackett, M. \& Miller, S. I. (1998). PmrA-PmrB-regulated genes necessary for 4aminoarabinose lipid A modification and polymyxin resistance. $\mathrm{Mol}$ Microbiol 27, 1171-1182.

Haagensen, J. A., Klausen, M., Ernst, R. K., Miller, S. I., Folkesson, A., Tolker-Nielsen, T. \& Molin, S. (2007). Differentiation and distribution of colistin- and sodium dodecyl sulfate-tolerant cells in Pseudomonas aeruginosa biofilms. J Bacteriol 189, 28-37.

Hiemstra, P. S., Fernie-King, B. A., McMichael, J., Lachmann, P. J. \& Sallenave, J. M. (2004). Antimicrobial peptides: mediators of innate immunity as templates for the development of novel anti-infective and immune therapeutics. Curr Pharm Des 10, 28912905.

Hoeprich, P. D. (1970). The polymyxins. Med Clin North Am 54, 1257-1265.

Høiby, N., Krogh Johansen, H., Moser, C., Song, Z., Ciofu, O. \& Kharazmi, A. (2001). Pseudomonas aeruginosa and the in vitro and in vivo biofilm mode of growth. Microbes Infect 3, 23-35.

Jacobs, M. A., Alwood, A., Thaipisuttikul, I., Spencer, D., Haugen, E., Ernst, S., Will, O., Kaul, R., Raymond, C. \& other authors (2003). Comprehensive transposon mutant library of Pseudomonas aeruginosa. Proc Natl Acad Sci U S A 100, 14339-14344.

Khandelia, H. \& Kaznessis, Y. N. (2005). Molecular dynamics simulations of helical antimicrobial peptides in SDS micelles: what do point mutations achieve? Peptides 26, 2037-2049.

King, J. D., Kocíncová, D., Westman, E. L. \& Lam, J. S. (2009). Review: lipopolysaccharide biosynthesis in Pseudomonas aeruginosa. Innate Immun 15, 261-312.

Koch, B., Jensen, L. E. \& Nybroe, O. (2001). A panel of Tn7-based vectors for insertion of the GFP marker gene or for delivery of cloned DNA into Gram-negative bacteria at a neutral chromosomal site. J Microbiol Methods 45, 187-195.

Lam, J., Matewish, M. \& Poon, K. K. H. (2004). Lipopolysaccharides of Pseudomonas aeruginosa. In Pseudomonas, vol. 3, Biosynthesis of Macromolecules and Molecular Metabolism, pp. 341-361. Edited by J. L. Ramos.

Lambertsen, L., Sternberg, C. \& Molin, S. (2004). Mini-Tn7 transposons for site-specific tagging of bacteria with fluorescent proteins. Environ Microbiol 6, 726-732.

Landman, D., Georgescu, C., Martin, D. A. \& Quale, J. (2008). Polymyxins revisited. Clin Microbiol Rev 21, 449-465.

Littlewood, J. M., Miller, M. G., Ghoneim, A. T. \& Ramsden, C. H. (1985). Nebulised colomycin for early pseudomonas colonisation in cystic fibrosis. Lancet 325, 865.

Manoil, C. (2000). Tagging exported proteins using Escherichia coli alkaline phosphatase gene fusions. Methods Enzymol 326, 35-47.

Matewish, M. (2004). The functional role of lipopolysaccharide in the cell envelope and surface proteins of Pseudomonas aeruginosa. $\mathrm{PhD}$ thesis, University of Guelph, Canada.

McPhee, J. B., Lewenza, S. \& Hancock, R. E. (2003). Cationic antimicrobial peptides activate a two-component regulatory system, PmrA-PmrB, that regulates resistance to polymyxin B and cationic antimicrobial peptides in Pseudomonas aeruginosa. Mol Microbiol 50, 205-217. 
Moskowitz, S. M., Ernst, R. K. \& Miller, S. I. (2004). PmrAB, a twocomponent regulatory system of Pseudomonas aeruginosa that modulates resistance to cationic antimicrobial peptides and addition of aminoarabinose to lipid A. J Bacteriol 186, 575-579.

Nickel, J. C., Ruseska, I., Wright, J. B. \& Costerton, J. W. (1985). Tobramycin resistance of Pseudomonas aeruginosa cells growing as a biofilm on urinary catheter material. Antimicrob Agents Chemother 27, 619-624.

Pamp, S. J., Gjermansen, M., Johansen, H. K. \& Tolker-Nielsen, T. (2008). Tolerance to the antimicrobial peptide colistin in Pseudomonas aeruginosa biofilms is linked to metabolically active cells, and depends on the pmr and mexAB-oprM genes. Mol Microbiol 68, 223-240.

Perron, G. G., Zasloff, M. \& Bell, G. (2006). Experimental evolution of resistance to an antimicrobial peptide. Proc Biol Sci 273, 251-256.

Prince, A. S. (2002). Biofilms, antimicrobial resistance, and airway infection. N Engl J Med 347, 1110-1111.

Sawai, M. V., Waring, A. J., Kearney, W. R., McCray, P. B., Jr, Forsyth, W. R., Lehrer, R. I. \& Tack, B. F. (2002). Impact of single-residue mutations on the structure and function of ovispirin/novispirin antimicrobial peptides. Protein Eng 15, 225-232.

Schneider, T., Kruse, T., Wimmer, R., Wiedemann, I., Sass, V., Pag, U., Jansen, A., Nielsen, A. K., Mygind, P. H. \& other authors (2010). Plectasin, a fungal defensin, targets the bacterial cell wall precursor Lipid II. Science 328, 1168-1172.

Southward, C. M. \& Surette, M. G. (2002). The dynamic microbe: green fluorescent protein brings bacteria to light. Mol Microbiol 45, 1191-1196.

Srinivas, N., Jetter, P., Ueberbacher, B. J., Werneburg, M., Zerbe, K., Steinmann, J., Van der Meijden, B., Bernardini, F., Lederer, A. \& other authors (2010). Peptidomimetic antibiotics target outer-membrane biogenesis in Pseudomonas aeruginosa. Science 327, 1010-1013.

Steinstraesser, L., Tack, B. F., Waring, A. J., Hong, T., Boo, L. M., Fan, M. H., Remick, D. I., Su, G. L., Lehrer, R. I. \& Wang, S. C. (2002). Activity of novispirin G10 against Pseudomonas aeruginosa in vitro and in infected burns. Antimicrob Agents Chemother 46, 1837-1844.
Storm, D. R., Rosenthal, K. S. \& Swanson, P. E. (1977). Polymyxin and related peptide antibiotics. Annu Rev Biochem 46, 723-763.

Travis, S. M., Anderson, N. N., Forsyth, W. R., Espiritu, C., Conway, B. D., Greenberg, E. P., McCray, P. B., Jr, Lehrer, R. I., Welsh, M. J. \& Tack, B. F. (2000). Bactericidal activity of mammalian cathelicidinderived peptides. Infect Immun 68, 2748-2755.

Trent, M. S., Ribeiro, A. A., Doerrler, W. T., Lin, S., Cotter, R. J. \& Raetz, C. R. (2001). Accumulation of a polyisoprene-linked amino sugar in polymyxin-resistant Salmonella typhimurium and Escherichia coli: structural characterization and transfer to lipid $\mathrm{A}$ in the periplasm. J Biol Chem 276, 43132-43144.

Vaara, M., Vaara, T., Jensen, M., Helander, I., Nurminen, M., Rietschel, E. T. \& Mäkelä, P. H. (1981). Characterization of the lipopolysaccharide from the polymyxin-resistant pmrA mutants of Salmonella typhimurium. FEBS Lett 129, 145-149.

Valdivia, R. H., Cirillo, D. M., Lee, A. K., Bouley, D. M. \& Falkow, S. (2000). mig-14 is a horizontally acquired, host-induced gene required for Salmonella enterica lethal infection in the murine model of typhoid fever. Infect Immun 68, 7126-7131.

Winfield, M. D. \& Groisman, E. A. (2004). Phenotypic differences between Salmonella and Escherichia coli resulting from the disparate regulation of homologous genes. Proc Natl Acad Sci U S A 101, 17162-17167.

Winsor, G. L., Van Rossum, T., Lo, R., Khaira, B., Whiteside, M. D., Hancock, R. E. \& Brinkman, F. S. (2009). Pseudomonas Genome Database: facilitating user-friendly, comprehensive comparisons of microbial genomes. Nucleic Acids Res 37 (Database issue), D483D488.

Zaslaver, A., Bren, A., Ronen, M., Itzkovitz, S., Kikoin, I., Shavit, S., Liebermeister, W., Surette, M. G. \& Alon, U. (2006). A comprehensive library of fluorescent transcriptional reporters for Escherichia coli. Nat Methods 3, 623-628.

Zasloff, M. (2002). Antimicrobial peptides of multicellular organisms. Nature 415, 389-395.

Edited by: W. J. Quax 